\title{
Avaliação morfológica de Pilocarpus microphyllus Stapf Ex Wardleworth
}

\author{
Morphologica evaluation of Pilocarpus microphyllus Stapf Ex Wardleworth \\ Evaluación morfológica de Pilocarpus microphyllus Stapf Ex Wardleworth
}

\section{Resumo}

Este trabalho objetivou avaliar acessos de jaborandi identificando os descritores morfológicos para a espécie. Foram avaliados 25 acessos, conservados in vivo na Embrapa Amazônia Oriental, em Belém, PA, totalizando 125 indivíduos, para oito caracteres morfológicos quantitativos. Os dados foram submetidos as analises multivariadas, realizado a seleção direta e seleção com reanálise agrupadas em dois métodos e com base na distância Euclidiana média utilizada na formação dos agrupamentos pelos métodos Unweighted Paired Group Method Using ArithmeticAverages UPGMA de otimização de Tocher e para análise de componentes principais. Dos oito caracteres avaliados segundo os dois procedimentos utilizados nenhum se mostrou redundante, logo não houve descarte. As dissimilaridades encontradas com base na distância euclidiana - média padronizada apresentaram três menores distâncias 0,$35 ; 0,44$ e 0,46 entre pares de acessos e permitiram separá-los em três grupos divergentes pelos métodos UPGMA e pelo método de Tocher houve a formação de quatro grupos distintos. Dois caracteres apresentaram as maiores contribuições para a divergência, sendo eles largura e comprimento da folha, com 35,4\%. Portanto, os acessos avaliados de jaborandi possuem variabilidade morfológica de acordo com os caracteres quantitativos: número de folíolos/folha, altura da planta; largura do folíolo, comprimento do pecíolo, comprimento da folha, largura da folha; comprimento do folíolo e comprimento da inflorescência, sendo os caracteres largura e comprimento da folha os que mais contribuíram para a divergência entre os acessos.

Palavras-chave: Jaborandi; Banco ativo de germoplasma; Caracteres; Variação morfológica.

\begin{abstract}
This work aimed to evaluate accessions of jaborandi, and to identify the morphological descriptors for the species. Twenty-five accessions, preserved in vivo at Embrapa Amazônia Oriental, in Belém, PA, were evaluated, totaling 125 individuals, for eight quantitative morphological characters. The data were submitted to multivariate analyzes, direct selection and selection with reanalysis were carried out grouped in two methods and based on the average Euclidean distance used in the formation of the clusters by the UPGMA methods of Tocher's optimization and for the analysis of main components. Of the eight characters evaluated according to the two procedures used, none was shown to be reduntant, so there was no discard. The dissimilarities found based on the Euclidean distance - standardized mean had three shortest distances $0.35 ; 0.44$ and 0.46 between pairs of accessions and allowed to separate them into three divergent groups by the Unweighted Paired Group Method Using ArithmeticAverages and by the Tocher method, there were the formation of four distinct groups. Two characters presented the greatest contributions to the divergence, being width and length of the leaf, with $35.4 \%$. Therefore, the evaluated accessions of jaborandi have morphological variability according to the quantitative characters: number of leaflets / leaf, plant height; leaflet width, petiole length, leaf length, leaf width; length of the leaflet and length of the inflorescence, the characters width and length of the leaf being the ones that most contributed to the divergence between the accessions.
\end{abstract}

Keywords: Jaborandi; Active germplasmbank; Characters; Morphological variation. 


\begin{abstract}
Resumen
Este trabajo tuvo como objetivo evaluar las accesiones de Jaborandi e identificar los descriptores morfológicos de la especie. Se evaluaron veinticinco accesiones, preservadas in vivo en Embrapa Amazônia Oriental, en Belém, PA, totalizando 125 individuos, para ocho caracteres morfológicos cuantitativos. Los datos se sometieron a análisis multivariados, la selección directa y el reanálisis se realizaron agrupados en dos métodos y con base en la distancia euclidiana promedio utilizada en la formación de los conglomerados por los métodos UPGMA de optimización de Tocher y para el análisis de componentes principales. De los ocho caracteres evaluados según los dos procedimientos utilizados, ninguno demostró ser redundante, por lo que no hubo descarte. Las diferencias encontradas con base en la distancia euclidiana: la media estandarizada tenía las tres distancias más cortas 0,35 ; 0,44 y 0,46 entre pares de accesiones y se les permitió separarlos en tres grupos divergentes mediante el método de grupos emparejados no ponderados utilizando promedios aritméticos y mediante el método de Tocher, se formaron cuatro grupos distintos. Dos personajes presentaron las mayores contribuciones a la divergencia, siendo el ancho y el largo de la hoja, con un $35,4 \%$. Por tanto, las accesiones evaluadas de jaborandi presentan variabilidad morfológica según los caracteres cuantitativos: número de folíolos / hoja, altura de planta; ancho del folíolo, largo del pecíolo, largo de la hoja, ancho de la hoja; la longitud del foliolo y la longitud de la inflorescencia, siendo el ancho de los caracteres y la longitud de la hoja los principales contribuyentes a la divergencia entre accesiones.
\end{abstract}

Palabras clave: Jaborandi; Banco de germoplasma activo; Caracteres; Variación morfológica.

\title{
1. Introdução
}

Existem várias espécies de plantas com propriedades medicinais identificadas no Brasil. Como exemplo dessa flora têm à espécie Pilocarpus microphyllus Stapf Ex Wardleworth, popularmente conhecida como jaborandi (Rocha et al., 2017). Segundo Silva (2018) o gênero Pilocarpus pertence à família das Rutaceaes encontra-se distribuído amplamente pela América do Sul e América Central, a maioria das espécies é nativa do Brasil.

Em meio às espécies de plantas medicinais produtoras de compostos bioativos de interesse mundial, o jaborandi é fonte de uma variedade de metabólitos secundários com potencial de atividade farmacológica, tais como alcalóides, cumarinas, flavonóides e terpenos (Santos; Moreno, 2004). Dessa forma sua importância econômica é devido ao fato de suas folhas serem fonte da pilocarpina.

O jaborandi é a fonte natural da pilocarpina, um alcalóide utilizado para tratar o glaucoma e xerostomia (Gil-Montoya et.al., 2016). Por apresentar essa propriedade o jaborandi foi fortemente explorado passando a ser considerada uma planta obrigatória em programas de conservação e incluído na lista da Convenção sobre o Comércio Internacional de Espécies da Flora e Fauna Selvagens em Perigo de Extinção, em conseqüência ao extrativismo desordenado realizado por folheiros que não seguiam nenhum método sustentável de coleta das folhas, arrancando o arbusto para retirar dele somente as folhas (ICMBio, 2009).

A vista disso, a Instituição Embrapa Amazônia Oriental localizada no município de Belém-PA,no ano de 1991 instalou o Banco Ativo de Germoplasma (Bag) de jaborandi com o objetivo de introduzir, coletar, conservar, caracterizar, documentar e disponibilizar o germoplasma dessa espécie (Pádua et al., 2020), nesse Bag os acessos coletados de diferentes procedências permitiu conduzir estudos na área de caracterização e avaliação morfológica.

Nesse sentido, Lameira et al., (2020) consideram que uma das tarefas mais importantes dentro dos programas de melhoramento é a conservação do germoplasma, pois serve como um reservatório de genes aos quais os melhoristas podem acessar quando precisam resolver problemas específicos, e for estudados em várias etapas como coleta ou introdução, multiplicação, preservação/ conservação, avaliação/ caracterização e uso racional.

Segundo Oliveira (2005) a conservação de indivíduos de interesse, pode ser feita em um Bag, que faz referência ao patrimônio genético de uma determinada espécie. Sendo portadores da variabilidade total que pode ser transmitida de uma geração para outra (Costa \& Spehar, 2012). Na avaliação de acessos em bancos de germoplasma a observação e avaliação de um grande número de características em um mesmo indivíduo são muito comuns, principalmente quando a quantidade de indivíduos é grande (Mingoti, 2005). 
Em função do exposto, este trabalho objetivou avaliar acessos de jaborandi, identificando os descritores morfológicos para a espécie.

\section{Metodologia}

O estudo foi realizado no Bag de jaborandi, instalado no Horto de Plantas Medicinais e Aromáticas da Embrapa Amazônia Oriental, com altitude de $10 \mathrm{~m}$ e temperatura média anual de $30^{\circ} \mathrm{C}$. O clima em Belém, segundo série histórica da cidade, apresenta precipitação anual média de $3.070 \mathrm{~mm}$, entretanto, as chuvas se distribuem de maneira diferente durante o ano, formando um período muito chuvoso (dezembro a maio) e um período menos chuvoso (junho a novembro) Lira et al., (2020).

Foram avaliados 25 acessos de jaborandi, conservados in vivo, cultivados a pleno sol, os tratos culturais e fitossanitários foram realizados de acordo com a necessidade da espécie. Os acessos possuem origem nos estados do Maranhão e Pará, sendo 15 acessos do Pará e 10 do estado do Maranhão, Tabela 1(Alelo vegetal, 2021). Foram selecionadas cinco plantas por acesso de forma aleatória e marcadas com fita. Este trabalho está de acordo com as definições sugeridas por Pereira et al., (2018), sendo uma pesquisa metodológica quantitativa.

Tabela 1. Denominações e procedências de 25 acessos conservados no Bag de jaborandi da Embrapa Amazônia Oriental.

\begin{tabular}{|c|c|c|}
\hline Código BAG & Código Local & Procedência \\
\hline Bonal 4 & BGAJB04 & Moju (PA) \\
\hline Bonal 3 & BGAJB03 & Moju (PA) \\
\hline Bonal 2 & BGAJB02 & Moju (PA) \\
\hline Bonal 1 & BGAJB01 & Moju (PA) \\
\hline NBB & BGAJNBB002 & Novo Breu Branco (PA) \\
\hline Maisa & BGAJMS001 & Moju (PA) \\
\hline Açailandia & BGAJ051 & Açailândia (MA) \\
\hline Bra-060 & BGAJ060 & Açailândia (MA) \\
\hline $\mathrm{CCM}$ & BGAJCCM01 & Moju (PA) \\
\hline Merk 1 & MK01 & Barra do Corda (MA) \\
\hline Japonês & BGAJJ003 & Moju (PA) \\
\hline Carajás 3 & BGAJSC03 & Parauapebas (PA) \\
\hline Carajás A & BGAJSC04 & Parauapebas (PA) \\
\hline Carajás B & BGAJSC05 & Parauapebas (PA) \\
\hline Carajás C & SC06 & Parauapebas (PA) \\
\hline Bra 132 & BGAJ132 & Nina Rodrigues (MA) \\
\hline Bra 159 & BGAJ159 & Mata Roma (MA) \\
\hline Bra 167 & BGAJ67 & Brejo (MA) \\
\hline N5W & N5W & Parauapebas (PA) \\
\hline Merk 2 & MK02 & Barra do Corda (MA) \\
\hline Merk 3 & MK03 & Barra do Corda (MA) \\
\hline Merk 4 & MK04 & Barra do Corda (MA) \\
\hline Merk 5 & MK05 & Barra do Corda (MA) \\
\hline Merk 6 & BGAJMK06 & Barra do Corda (MA) \\
\hline Merk 7 & SC07 & Parauapebas (PA) \\
\hline
\end{tabular}


Para seleção de descritores e divergência genética entre os acessos os caracteres morfológicos quantitativos avaliados foram: número de folíolos/folha, altura da planta; largura do folíolo, comprimento do pecíolo, comprimento da folha, largura da folha; comprimento do folíolo e comprimento da inflorescência. A unidade de medição para todas as variáveis foi em centímetros (cm) e extraídos com o auxílio de régua e paquímetro.

Após tabulação dos dados, foram realizados dois procedimentos com o auxílio estatístico do software R (2020) para identificação de possíveis caracteres redundantes ou invariáveis para posterior seleção dos descritores. O primeiro procedimento foi de seleção direta, proposto por Jolliffe (1937) neste caso, foram eliminados todos os caracteres que apresentaram maior coeficiente de ponderação em valor absoluto (autovetor) no componente principal de menor autovalor, partindo do último componente até aquele cujo autovalor não excedesse a 0,70. O segundo foi da seleção com reanálise, sugerida por Cury (1993) onde cada caráter sugerido para descarte, é realizado nova análise com os caracteres remanescentes, examinando os coeficientes de correlação do caráter sugerido para descarte com os demais caracteres, sendo finalizada quando o caráter a ser descartado se mostrou altamente correlacionado com pelo menos um caráter já descartado. O descarte final é realizado com base na informação obtida simultaneamente nos dois procedimentos.

Os dados dos descritores selecionados foram empregados para a obtenção das dissimilaridades entre cada par de acessos com base na distância euclidiana média padronizada, no mesmo software estatístico. Uma vez que os acessos encontram-se estabelecidos sem obedecer a nenhum delineamento experimental, assim como os caracteres em diferentes medidas e escalas, esta análise foi executada com base na média de cada caráter.

A matriz de distâncias obtidas foi utilizada na formação dos agrupamentos pelos métodos de UPGMA, otimização de Tocher e para análise de componentes principais.

\section{Resultados e Discussão}

Dos oito caracteres avaliados segundo os dois procedimentos utilizados nenhum se mostrou redundante, logo não houve descarte. Assim, a eliminação de descritores redundantes é de grande importância, pois minimiza os esforços na avaliação de genótipos sem perda na precisão de avaliação dos genótipos (Albuquerque \& Leite, 2020), especialmente se esses caracteres forem de difícil mensuração e apresentarem baixa variabilidade (Daher, 1993). Por isso, neste trabalho foi realizado os procedimentos de seleção direta, proposto por Jolliffe (1937) e a seleção com reanálise, sugerida por Cury (1993), entretanto os testes não identificaram redundância que justifiquem possíveis descartes. Brandão et al., (2013) ao propor um número mínimo de descritores morfoagronômicos capaz de quantificar a divergência genética entre acessos de bananeira eliminou 20 dos 27 caracteres quantitativos através do método direto proposto por Jolliffe (1973).

A matriz de dissimilaridade obtida entre os pares de 25 acessos de jaborandi gerada com base em oito caracteres quantitativos revela maior semelhança, ou seja, maior similaridade e menor distância $(0,35)$ entre os acessos Carajás $\mathrm{B}$ x Carajás 3 ambos provenientes do Estado do Pará (PA). A segunda menor distância $(0,44)$ entre os pares, Merk 2 x Bra 132 ambos provenientes do Estado do Maranhão (MA) e a terceira menor distância $(0,46)$ entre os pares Merk5 (MA) x N5W (PA). Costa et al., (2006) relatam que a identificação dos diferentes graus de divergência genética é importante uma vez que fornece parâmetros para a identificação de genitores promissores.

Com base no método UPGMA os 25 acessos formaram três grupos divergentes: o grupo I contendo o acesso Maisa coletados no PA, o II composto pelos acessos Merck1 (MA) e Bonal 3 (PA) e III contendo os demais acessos com diferentes localidades (Figura 1). Pode-se perceber que apesar de diferentes procedências, houve o agrupamento destes acessos, nos grupos II e III. O aumento da distância geográfica nem sempre implica em maior divergência genética, pois a deriva genética e a seleção em diferentes ambientes podem contribuir muito mais para a divergência do que a separação geográfica (Dias,1994), 
isto corrobora que nem sempre a diversidade geográfica é sinônimo de divergência genética conforme ressaltam Cruz e Carneiro (2006).

A formação de um número razoável de grupos em um dendograma demonstra a existência da variabilidade genética entre os acessos avaliados, uma vez que a formação de poucos grupos representa uma distância genética baixa (Martins, 2011).

Figura 1. Dendograma gerado pelo método UPGMA a partir das dissimilaridades obtidas entre 25 acessos de jaborandi, conservados no bag da Embrapa Amazônia Oriental, com base em oito caracteres morfológicos.

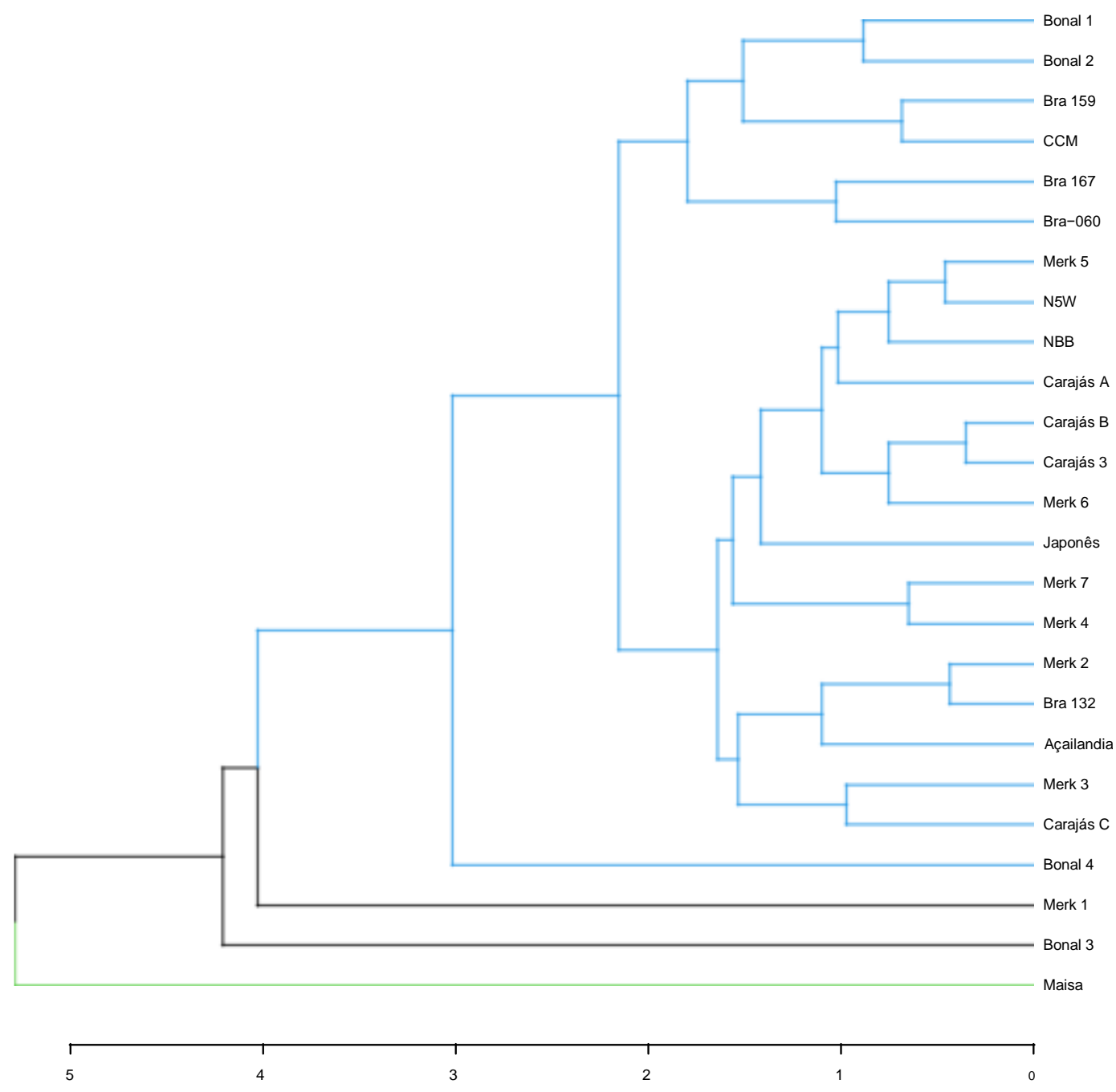

Fonte: Autores.

Pelo método de Tocher houve a formação de quatro grupos divergentes entre os 25 acessos (Tabela 2). No grupo I esta presente apenas o acesso Bonal 3, o grupo II contém o acesso Maisa (PA), o grupo III agrupa o acesso Merck1, os demais acessos formam o grupo IV. Observou-se alguma semelhança na formação dos grupos com a análise pelo método do UPGMA, ressalta-se a formação de um grupo contendo unicamente o acesso Maisa. Como são acessos da mesma espécie que formam grupos distintos reflete o grau de divergência genética encontrado entre eles e a importância de serem conservados no banco de germoplasma, a fim de se manter a acentuada variabilidade entre os materiais (Dattatrayet al.,2006).Diante disso, o método de 
otimização de Tocher aliado ao método UPGMA, confere maior eficiência em discriminar os acessos quanto as suas distâncias genéticas (Nardino et al., 2017).

Tabela 2. Formação dos grupos divergentes pelo método de Tocher entre os 25 acessos de jaborandi conservados no bag da Embrapa Amazônia Oriental, com base em oito caracteres morfológicos.

\begin{tabular}{llllllll}
\hline Grupos & Acessos & & & & & & \\
\hline I & Bonal 3 & & & & & & \\
II & Maisa & & & & & & \\
III & Merk 1 & & & & & & \\
IV & Carajás 3 & Carajás B & Merk 5 & N5W & Merk 6 & Carajás A & NBB \\
& Merk 2 & Bra 132 & Merk 3 & Carajás C & Merk 7 & Merk 4 & Bra 167 \\
& Bonal 2 & Bonal 1 & Japonęs & Açailandia & CCM & Bra 159 & Bra-060 \\
& Bonal 4 & & & & & & \\
\hline
\end{tabular}

Fonte: Autores.

A largura da folha e comprimento da folha foram os caracteres que mais contribuíram para a divergência entre os acessos estudados com percentuais de contribuição igual a 18,89 \% e 16,50 \%, respectivamente, (Tabela 3). Resultados semelhantes foram observados por Lameira et al., (2020), realizando avaliações de componente principais para avaliação de jaborandi, notou maior contribuição relativa para as divergências genotípicas encontradas.

O componente que menos contribuiu foi o caráter comprimento da inflorescência com 5,24\%. A análise de componentes principais vem se destacando como a metodologia mais empregada em bancos e ou coleções de germoplasma, pois além de identificar os caracteres mais importantes na contribuição da divergência genética (Pereira, 1999), pois são responsáveis por direções que estão associadas à baixa variabilidade (Hongyu et al., 2016). 
Tabela 3. Contribuição relativa para a dissimilaridade de 25 acessos de jaborandi conservados no bag da Embrapa Amazônia Oriental, com base em oito caracteres morfológicos.

\begin{tabular}{ll}
\hline Caráter & Contribuição (\%) \\
\hline Número de folíolo/ folha & 12.9 \\
Altura da planta & 12.9 \\
Largura do folíolo & 8.5 \\
Comprimento do pecíolo & 12,0 \\
Comprimento da folha & 16.5 \\
Largura da folha & 18.9 \\
Comprimento do folíolo central & 13.1 \\
Tamanho da inflorescência & 5.2 \\
\hline
\end{tabular}

Fonte: Autores.

A análise conjunta de dados qualitativos e quantitativos é viável e pode permitir maior eficiência no conhecimento da divergência entre acessos de espécies vegetais conservadas em bancos de germoplasma (Machado et al., 2015). Igualmente Lameira et al., (2020) avaliando descritores morfoagronômico para a espécie jaborandi verificaram a possibilidade de utilização de caracteres qualitativos para diferenciação entre os acessos estudados.

\section{Conclusão}

Este estudo avaliou a variabilidade dos caracteres morfológicos quantitativos: número de folíolos/folha, altura da planta; largura do folíolo, comprimento do pecíolo, comprimento da folha, largura da folha; comprimento do folíolo e da inflorescência, todos apresentaram contribuição para diferenciação entre os acessos avaliados de jaborandi.

Identificou e agrupou os descritores; os que mais contribuíram para a divergência entre os acessos foram largura da folha e comprimento da folha, mostrando-se importantes na avaliação do germoplasma de jaborandi.

Recomenda-se a soma de estudos de caracteres morfológicos qualitativos para a espécie. Estas informações são cruciais para a espécie, pois auxilia no conhecimento e uso da variabilidade genética e podem servir de base para a seleção de indivíduos de interesse dos programas de melhoramento, além de fornecer informações fundamentais que facilitará a identificação de espécimes em ecossistemas nativos ou plantados.

\section{Agradecimentos}

Os autores agradecem à Coordenação de Aperfeiçoamento de Pessoal de Nível Superior (CAPES) pelo apoio e bolsa de estudo de Doutorado, à Embrapa Amazônia Oriental por ceder o local e a estrutura, e ao Programa de Doutorado da Rede Bionorte- Biodiversidade e Biotecnologia.

\section{Referências}

Albuquerque, D. P., \& Leite, F. D. S. (2020). Dissimilaridade genética e seleção de descritores da parte aérea de jambu [Acmella oleracea (L.) RK Jansen].

Arriel, N. H. C., Dos Santos, J. W., Moreira, J. D. A. N., Nóbrega, M. B. D. M., \& De Andrade, F. P. (2000). Avaliação de descritores quantitativos na caracterização preliminar de germoplasma de gergelim (Sesamum indicum L.) 1. Revista Brasileira de Oleaginosas e Fibrosas, 4(1).

Brandão, L. P., Souza, C. P. F., Pereira, V. M., Silva, S. O., Santos-Serejo, J. A., Ledo, C. A. S., \& Amorim, E. P. (2013). Descriptor selection for banana accessions based on univariate and multivariate analysis. Genetics and Molecular Research, 12(2), 1603-1620.

Costa, A. M., Spehar, C. R., \& Sereno, J. R. B. (2012). Conservação de recursos genéticos no Brasil. Embrapa Cerrados-Livro científico (ALICE). 
Costa, M. N. D., Pereira, W. E., Bruno, R. D. L. A., Freire, E. C., Nóbrega, M. B. D. M., Milani, M., \& Oliveira, A. P. D. (2006). Divergência genética entre acessos e cultivares de mamoneira por meio de estatística multivariada. Pesquisa Agropecuária Brasileira, 41(11), 1617-1622.

Cruz, C. D., \& Souza Carneiro, P. C. (2006). Modelos biométricos aplicados ao melhoramiento genético (No. 575.1015195). Universidad Federal de Viçosa.

Cury, R. (1993). Dinâmica evolutiva e caracterizaçao de germoplasma de mandioca (Manihot esculenta, Crantz) na agricultura autóctone do Sul do Estado de Sao Paulo (Doctoral dissertation, Universidade de São Paulo).

Daher, R. (1993). Diversidade morfológica e isoenzimática em capim elefante (Pennisetum purpureum Schum.).110 f (Doctoral dissertation, Dissertação (Mestrado em Genética e Melhoramento de Plantas)-Universidade Federal de Viçosa, Viçosa, MG).

Dattatraya, A.; Rankin, S. A. (2006) O PH moderadamente ácido potencializa o escurecimento do soro de leite doce em pó. International Dairy Journal,16 (7), $822-828$.

Dias, L. A. D. S. (1994). Divergência genética e fenética multivariada na predição de híbridos e preservação de germoplasma de cacau (Theobroma cacao L.) (Doctoral dissertation, Universidade de São Paulo).

Embrapa. (2021). Embrapa Recursos Genéticos E Biotecnologia. Núcleo de Tecnologia da Informação (NTI). AleloVegetal.

Gil-Montoya, J. A., Silvestre, F. J., Barrios, R., \& Silvestre-Rangil, J. (2016). Treatment of xerostomia and hyposalivation in the elderly: A systematic review. Medicina oral, patologia oral y cirugia bucal, 21(3), e355.

Hongyu, K., Sandanielo, V. L. M., \& de Oliveira Junior, G. J. (2016). Análise de componentes principais: resumo teórico, aplicação e interpretação. E\&S Engineering and Science, 5(1), 83-90.

ICMBio - www.icmbio.gov.br 14/01/2009 http://ti.socioambiental.org/\#!/noticia/63448.

Jolliffe, I. T. (1973). Discarding variables in a principal componentanalysis. II: Real data. Journal of the Royal Statistical Society: Series C (AppliedStatistics), 22(1), 21-31.

Lameira, O. A., Cordeiro, I. M. C. C., \& Pires, H. C. G. (2020). Avaliação dos Descritores Morfoagronômico e Morfoanatomia da Lâmina Foliar de Pilocarpus: Microphyllus Stapf ex Wardleworth-Rutaceae, Ananas Comosus Var. Erectifolius (LB Smith) Coppens \& F. Leal-Bromeliacea e Psychotria Ipecacuanha (Brot.) Stokes. Editora Appris.

Lira, B. R. P., Lopes, L. D. N. A., das Chaves, J. R., Santana, L. R., \& Fernandes, L. L. (2020). Identificação de Homogeneidade, Tendência e Magnitude da Precipitação em Belém (Pará) entre 1968 e 2018. Anuário do Instituto de Geociências, 43(4), 426-439.

Machado, C. D. F., Jesus, F. N. D., \& Ledo, C. A. D. S. (2015). Divergência genética de acessos de maracujá utilizando descritores quantitativos e qualitativos. Revista Brasileira de Fruticultura, 37(2), 442-449.

Martins, F. A. (2011). Integração de dados morfoagronômicos, moleculares e fitopatológicos para estabelecimento de coleção nuclear.

Mingoti, S. A. (2005). Análise de dados através de métodos de estatística multivariada: uma abordagem aplicada. UFMG.

Nardino, M., Baretta, D., Carvalho, I. R., Follmann, D. N., Ferrari, M., de Pelegrin, A. J., \& de Souza, V. Q. (2017). Divergência genética entre genótipos de milho (Zea mays L.) em ambientes distintos. Revista de Ciências Agrárias, 40(1), 164-174.

Oliveira, M. do S. P. (2005). Caracterização Molecular e Morfo-agronômica de germoplasma de açaizeiro. Tese (Doutorado) - (Universidade Federal de Lavras, Lavras.

Pádua, J. G., Albuquerque, M., \& de Mello, S. C. M. (2020). Bancos e coleções de germoplasma da Embrapa: Conservação e uso. Embrapa Recursos Genéticos e Biotecnologia-Documentos (INFOTECA-E).

Pereira V .A. (1999). Utilização de análise multivariada na caracterização de germoplasma de mandioca ( ManihotesculentaCrantz.) [tese]. Piracicaba: Escola Superior de Agricultura Luiz de Queiroz, Universidade de São Paulo.

Pereira, A. S.; Shitsuka, D. M.; Parreira, F. J. \& Shitsuka, R. (2018). Metodologia da pesquisa científica. UFSM.

R Core Team (2020). R: A languageandenvironment for statisticalcomputing. R Foundation for StatisticalComputing, qaw13q .

Rocha, J. A., Andrade, I. M., Véras, L. M., Quelemes, P. V., Lima, D. F., Soares, M. J., \& Leite, J. R. (2017). Anthelmintic, antibacterial and cytotoxicity activity of imidazole alkaloids from Pilocarpus microphyllus leaves. Phytotherapy research, 31(4), 624-630.

Santos, A. P., \& Moreno, P. R. H. (2004). Pilocarpus spp.: A survey of its chemical constituents and biological activities. Revista brasileira de ciências farmacêuticas, 40(2), 116-137.

Silva, J. C. D. (2018). Identificação de Compostose Avaliação citotóxica, antibacteriana, antioxidante e antifúngico do óleo essencial de Pilocarpus microphyllus. 